\title{
Predictors for Reintervention after Surgical Coarctation Repair through Left Thoracotomy
}

\author{
Ayman R Abdelrehim ${ }^{1,2}$, Luna S Baangood ${ }^{3}$, Saad Q Khoshhal ${ }^{3}$, Rafik F.B. Soliman*1 \\ ${ }^{1}$ Cardiothoracic Surgery Department, Faculty of Medicine, Menoufia University, Menoufia, Egypt. \\ ${ }^{2}$ Cardiac Surgery Department, Madina Cardiac Center, Madina, Saudi Arabia \\ ${ }^{3}$ Pediatric Cardiology Department, Madina Cardiac Center, Madina, Saudi Arabia \\ *Corresponding author: Rafik F.B. Soliman, Mobile: (+20) 01011102102, E-Mail: rafikfekry@ hotmail.com
}

\begin{abstract}
Background: Recoarctation of the aorta may occur in infants after the primary surgical repair. Several risk factors were suggested in the literature, with controversial results among the studies.

Objective: This study was conducted to evaluate the predictors for reintervention after surgical coarctation repair by different techniques through left thoracotomy.

Patients and Methods: A retrospective analysis was performed for patients who underwent isolated surgical coarctation repair or with pulmonary artery band between Sep 2014 and December 2018. All primary intervention was through thoracotomy. Reintervention was defined as the need for balloon angioplasty or reoperation.

Results: A total of 47 patients with median age at repair of 7 days. The median duration of follow-up was 18 months (95\% CI=14 to 22 months). Only 7 (14.9\%) patients required reintervention. No mortality was recorded during the follow-up period. Cox proportional hazard analysis showed that only postoperative pressure gradient was an independent risk factor for reintervention. Receiver operating characteristic curve showed that postoperative pressure gradient was an excellent indicator (AUC $=0.948,95 \%$ CI: 0.841 to $0.992, p<0.001$ ), with the optimal cut-off value $>25$, sensitivity of $100 \%$ and specificity of $80 \%$.

Conclusions: Repair of aortic coarctation through thoracotomy has an overall good outcome. However, reintervention is required in some patients and elevated pressure gradient on echocardiographic assessment $<25$ can exclude recoarctation.
\end{abstract}

Keywords: Aortic coarctation, Infant, Outcome, Recurrence, Risk factors.

\section{INTRODUCTION}

Aortic coarctation (CoA) is a congenital anomaly that accounts for $6-8 \%$ of all cardiac anomalies, and it is the fourth most common heart defect requiring intervention in young infants ${ }^{(\mathbf{1 , 2})}$. The condition is characterized by narrowing of the proximal thoracic aorta near the ductus arteriosus, which in some cases may amount to long segment stenosis and arch hypoplasia ${ }^{(3,4)}$. It may develop as an isolated congenital anomaly or occurs in association with other cardiac anomalies such as septal defects, patent ductus arteriosus, and transposition of the great arteries ${ }^{(5,6)}$.

Patients with CoA may be present during or after the neonatal or breastfeeding period. The manifestations of ductal-dependent cases during the neonatal period are due to the closure of the ductus arteriosus, which manifests itself in the form of respiratory distress, heart failure and shock. Early intervention is required to improve patients' outcomes ${ }^{(7,8)}$.

Surgical repair of CoA aims to achieve a durable repair and avoid morbidity ${ }^{(9)}$. Several surgical approaches now are available for repair of CoA, including resection and simple end-to-end anastomosis, patch aortoplasty, subclavian flap aortoplasty, and resection with extended end-to-end anastomosis. However, recoarctation necessitating reintervention occurs with varying rates following the primary repair (10-12)
Several potential risk factors for aortic recoarctation and reintervention were studied, with

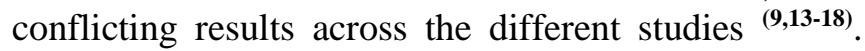
Therefore, the present study aimed to evaluate the rate and predictors for reintervention after surgical coarctation repair by different techniques through left thoracotomy.

\section{PATIENTS AND METHODS}

This retrospective cohort study was conducted by reviewing files of patients who underwent repair of $\mathrm{CoA}$ during the period from Sep 2014 and December 2018 at MCC Madina Cardiac Center, Saudia Arabia and El Aml Hospital, Cairo, Egypt.

\section{Ethical approval:}

The study was approved by the ethical committee of the Madina Cardiac Center and El Aml Hospital.

Confidentiality of patients' information was maintained by keeping the data collection sheet and the datasheet anonymous after assigning a code to each patient.

\section{Eligibility criteria:}

All patients who underwent primary repair of CoA in the first 3 months of life through thoracotomy. Patients with complex congenital heart diseases correction were excluded.

This article is an open access article distributed under the terms and conditions of the Creative Commons Attribution (CC BY-SA) license (http://creativecommons.org/licenses/by/4.0/) 


\section{Data collection:}

Patients' characteristics including age, sex, birth weight, as well as weight and height at surgery were collected. The files were also revised for presenting clinical manifestations, preoperative use of prostaglandin $\mathrm{E}$ and/or inotropes, and preoperative echocardiographic assessment. Collected operative details included the type of surgical procedure, pulmonary artery banding, and the cross-clamp time. Postoperative data were also collected comprising the duration of follow-up, admission into pediatric ICU, use of inotropes and duration of mechanical ventilation, postoperative complications as well as echocardiographic assessment before discharge and at follow-up.

\section{Statistical methods}

The analysis was conducted using Statistical Package for the Social Sciences for Windows, version 26 (IBM Corp., Armonk, N.Y., USA). Distribution of numerical variables was assessed using the ShapiroWilk test for normality. Normally distributed numerical variables were summarized as mean \pm standard deviation (SD), and comparisons between two groups were tested by independent samples T-test. Abnormally distributed numerical variables were summarized as median and interquartile range (IQR; $25^{\text {th }}-75^{\text {th }}$ percentiles), and comparisons were done using MannWhitney test.

Categorical variables were presented as frequencies and associations between two categorical variables were tested using Pearson's Chi square test, Fisher's exact test or Fisher-Freeman-Halton exact test as indicated. Survival analysis with Kaplan-Meier curve was used to calculate the median follow-up period and the rate of freedom from reintervention. Cox proportional hazard regression analysis was carried out to identify potential independent risk factors for reintervention, and hazard ratios (HR) and their 95\% confidence interval (CI) were calculated. Receiver operating characteristic (ROC) curve was performed for identified continuous risk factors. A p-value $<0.05$ was chosen to interpret significance of statistical tests.

\section{RESULTS}

The present study included 47 patients with coarctation of the aorta. Patients were categorized according to their need for reintervention (catheter angiographic-guided balloon dilatation) into two groups. Reintervention was required in 7 (14.9\%) patients. All reinterventions were performed in the first year following the primary repair, with a rate of freedom from reintervention of $83.9 \%$ at 12 months.

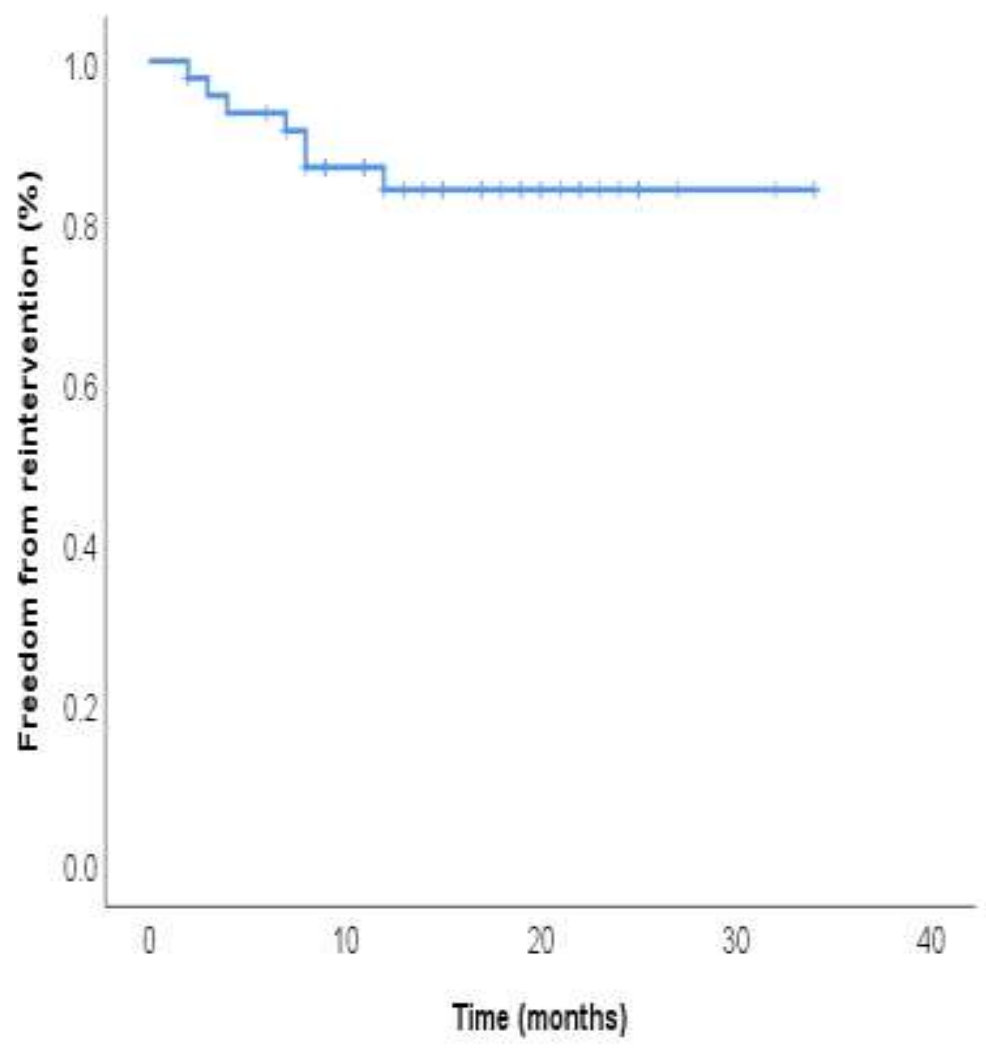

Figure (1): Kaplan-Meier curve for freedom from reintervention

Table (1) depicts preoperative characteristics of the included patients. No significant differences were detected in preoperative characteristics between the reintervention and no-reintervention groups, except that the reintervention group had a significantly higher percentage of low-birth-weight patients and all patients were administered prostaglandins preoperatively. 
Table (1): Patients' characteristics

\begin{tabular}{|c|c|c|c|c|c|c|c|}
\hline & \multicolumn{2}{|c|}{$\begin{array}{c}\text { Total } \\
(n=47)\end{array}$} & \multicolumn{2}{|c|}{$\begin{array}{c}\text { No reintervention } \\
(n=40)\end{array}$} & \multicolumn{2}{|c|}{$\begin{array}{c}\text { Reintervention } \\
\quad(\mathbf{n}=7)\end{array}$} & $\mathbf{P}$ \\
\hline \multicolumn{8}{|l|}{ Age (days) } \\
\hline Median [IQR] & \multicolumn{2}{|c|}{$7.0[1.0-14.0]$} & \multicolumn{2}{|c|}{$7.5[1.5-15.5]$} & \multicolumn{2}{|c|}{$6.0[1.0-14.0]$} & 0.420 \\
\hline Range & & $-90.0)$ & & $0-90.0)$ & & $-14.0)$ & \\
\hline \multicolumn{8}{|l|}{ Gender } \\
\hline Male & 26 & $55.3 \%$ & 21 & $52.5 \%$ & 5 & $71.4 \%$ & \multirow[t]{2}{*}{0.436} \\
\hline Female & 21 & $44.7 \%$ & 19 & $47.5 \%$ & 2 & $28.6 \%$ & \\
\hline \multicolumn{8}{|l|}{ Body weight at birth (kg) } \\
\hline Mean \pm SD & \multirow{2}{*}{\multicolumn{2}{|c|}{$\begin{array}{c}2.7 \pm 0.4 \\
(1.8-3.7)\end{array}$}} & \multirow{2}{*}{\multicolumn{2}{|c|}{$\begin{array}{c}2.8 \pm 0.4 \\
(1.8-3.7)\end{array}$}} & \multirow{2}{*}{\multicolumn{2}{|c|}{$\begin{array}{c}2.5 \pm 0.3 \\
(2.1-3.0)\end{array}$}} & 0.066 \\
\hline Range & & & & & & & \\
\hline \multicolumn{8}{|l|}{ Birth weight $<2.5 \mathrm{Kg}$} \\
\hline & 10 & $(21.3 \%)$ & 6 & $(15.0 \%)$ & 4 & $(57.1 \%)$ & $0.029 *$ \\
\hline \multicolumn{8}{|c|}{ Body weight $<2.5 \mathrm{Kg}$ at operation } \\
\hline & 10 & $(21.3 \%)$ & 9 & $(22.5 \%)$ & 1 & $(14.3 \%)$ & 1.000 \\
\hline \multicolumn{8}{|l|}{ Height (cm) } \\
\hline Mean \pm SD & \multirow{2}{*}{\multicolumn{2}{|c|}{$\begin{array}{c}50.6 \pm 4.6 \\
(45.0-67.0)\end{array}$}} & \multirow{2}{*}{\multicolumn{2}{|c|}{$\begin{array}{c}50.8 \pm 5.0 \\
(45.0-67.0)\end{array}$}} & \multirow{2}{*}{\multicolumn{2}{|c|}{$\begin{array}{c}49.7 \pm 1.0 \\
(48.0-51.0)\end{array}$}} & 0.568 \\
\hline Range & & & & & & & \\
\hline \multicolumn{8}{|l|}{ Body weight at time of surgery (g) } \\
\hline Mean \pm SD & & \pm 0.7 & & $0 \pm 0.8$ & & $7 \pm 0.2$ & 0.341 \\
\hline Range & & $-6.0)$ & & $1-6.0)$ & & $4-2.9)$ & \\
\hline PICU Admission & & & & & & & \\
\hline & 43 & $91.5 \%$ & 36 & $90.0 \%$ & 7 & $100.0 \%$ & 1.000 \\
\hline Clinical manifestations & & & & & & & \\
\hline Asymptomatic & 4 & $8.5 \%$ & 3 & $7.5 \%$ & 1 & $14.3 \%$ & 0.488 \\
\hline Cyanosis & 16 & $34.0 \%$ & 12 & $30.0 \%$ & 4 & $57.1 \%$ & 0.208 \\
\hline Respiratory distress & 35 & $74.5 \%$ & 30 & $75.0 \%$ & 5 & $71.4 \%$ & 1.000 \\
\hline Poor feeding & 7 & $14.9 \%$ & 6 & $15.0 \%$ & 1 & $14.3 \%$ & 1.000 \\
\hline Decreased activity & 1 & $2.1 \%$ & 1 & $2.5 \%$ & 0 & $0.0 \%$ & 1.000 \\
\hline Murmur & 4 & $8.5 \%$ & 3 & $7.5 \%$ & 1 & $14.3 \%$ & 0.488 \\
\hline Cardiogenic shock & 3 & $6.4 \%$ & 3 & $7.5 \%$ & 0 & $0.0 \%$ & 1.000 \\
\hline Preoperative shock & 10 & $21.3 \%$ & 7 & $17.5 \%$ & 3 & $42.9 \%$ & 0.155 \\
\hline Preoperative renal impairment & 3 & $6.4 \%$ & 3 & $7.5 \%$ & 0 & $0.0 \%$ & 1.000 \\
\hline Simple/complex cardiac anato & & & & & & & \\
\hline Simple & 40 & $85.1 \%$ & 35 & $87.5 \%$ & 5 & $71.4 \%$ & 0.276 \\
\hline Complex & 7 & $14.9 \%$ & 5 & $12.5 \%$ & 2 & $28.6 \%$ & \\
\hline Associated cardiac conditions & & & & & & & \\
\hline Yes & 33 & $70.2 \%$ & 27 & $67.5 \%$ & 6 & $85.7 \%$ & 0.657 \\
\hline ASD & 9 & $19.1 \%$ & 6 & $15.0 \%$ & 3 & $42.9 \%$ & 0.117 \\
\hline VSD & 22 & $46.8 \%$ & 18 & $45.0 \%$ & 4 & $57.1 \%$ & 0.690 \\
\hline DTGA & 4 & $8.5 \%$ & 2 & $5.0 \%$ & 2 & $28.6 \%$ & 0.100 \\
\hline DORV & 3 & $6.4 \%$ & 2 & $5.0 \%$ & 1 & $14.3 \%$ & 0.391 \\
\hline Transverse arch hypoplasia & 17 & $36.2 \%$ & 12 & $30.0 \%$ & 5 & $71.4 \%$ & 0.081 \\
\hline PDA & 31 & $66.0 \%$ & 28 & $70.0 \%$ & 3 & $42.9 \%$ & 0.208 \\
\hline Others & 9 & $19.1 \%$ & 8 & $20.0 \%$ & 1 & $14.3 \%$ & 1.000 \\
\hline Associated non-cardiac condit & & & & & & & \\
\hline & 19 & $40.4 \%$ & 16 & $40.0 \%$ & 3 & $42.9 \%$ & 1.000 \\
\hline Ventilation & & & & & & & \\
\hline & 11 & $23.4 \%$ & 8 & $20.0 \%$ & 3 & $42.9 \%$ & 0.330 \\
\hline Inotropes & 11 & $23.4 \%$ & 11 & $27.5 \%$ & 0 & $0.0 \%$ & 0.175 \\
\hline Prostaglandin & & & & & & & \\
\hline & 24 & $51.1 \%$ & 17 & $42.5 \%$ & 7 & $100.0 \%$ & $0.009^{*}$ \\
\hline
\end{tabular}

IQR: interquartile range; SD: standard deviation; *: significant difference. 
Table (2) demonstrates data of cardiovascular examination and echocardiography in the studied patients. There was no statistically significant difference between the two groups.

Table (2): Cardiovascular examination and echocardiography

\begin{tabular}{|c|c|c|c|c|c|}
\hline & \multicolumn{2}{|c|}{$\begin{array}{c}\text { Total } \\
(n=47)\end{array}$} & $\begin{array}{l}\text { No reintervention } \\
\quad(n=40)\end{array}$ & $\begin{array}{c}\text { Reintervention } \\
\quad(\mathbf{n}=7)\end{array}$ & $\mathbf{P}$ \\
\hline \multicolumn{6}{|c|}{ Preoperative U/L BP difference } \\
\hline Median [IQR] & \multirow{2}{*}{\multicolumn{2}{|c|}{$\begin{array}{l}20.0[15.0-40.0] \\
\quad(5.0-70.0)\end{array}$}} & \multirow{2}{*}{$\begin{array}{c}25.0[14.0-40.0] \\
(5.0-70.0)\end{array}$} & \multirow{2}{*}{$\begin{array}{c}20.0[20.0-45.0] \\
(5.0-50.0)\end{array}$} & \multirow{2}{*}{0.895} \\
\hline Range & & & & & \\
\hline \multicolumn{6}{|l|}{ Preoperative LV function } \\
\hline Poor & 8 & $17.0 \%$ & $15.0 \%$ & $28.6 \%$ & \multirow{3}{*}{0.533} \\
\hline Moderate & 11 & $23.4 \%$ & $22.5 \%$ & $28.6 \%$ & \\
\hline Good & 28 & $59.6 \%$ & $62.5 \%$ & $42.9 \%$ & \\
\hline \multicolumn{6}{|l|}{ Preoperative COA PG (ECHO) } \\
\hline Mean \pm SD & \multirow{2}{*}{\multicolumn{2}{|c|}{$\begin{array}{c}52.5 \pm 12.4 \\
(30.0-90.0)\end{array}$}} & $51.6 \pm 12.8$ & \multirow{2}{*}{$\begin{array}{c}57.6 \pm 8.9 \\
(48.0-75.0)\end{array}$} & \multirow[t]{2}{*}{0.244} \\
\hline Range & & & $(30.0-90.0)$ & & \\
\hline
\end{tabular}

IQR: interquartile range; SD: standard deviation; *: significant difference.

Table (3) depicts the operative data of the studied patients. There were no significant differences between the two groups.

Table (3): Operative details

\begin{tabular}{|c|c|c|c|c|c|c|c|}
\hline \multirow{2}{*}{ Type of COA Surgery } & \multicolumn{2}{|c|}{$\begin{array}{c}\text { Total } \\
(n=47)\end{array}$} & \multicolumn{2}{|c|}{$\begin{array}{l}\text { o reintervention } \\
\quad(\mathbf{n}=\mathbf{4 0})\end{array}$} & \multicolumn{2}{|c|}{$\begin{array}{l}\text { Reintervention } \\
\quad(\mathbf{n}=7)\end{array}$} & \multirow[t]{2}{*}{$\mathbf{P}$} \\
\hline & & & & & & & \\
\hline $\begin{array}{l}\text { Arch hypoplasia repair with interposition } \\
\text { graft }\end{array}$ & 1 & $2.1 \%$ & 0 & $0.0 \%$ & 1 & $14.3 \%$ & 0.149 \\
\hline Extended end to end anastomosis & 12 & $25.5 \%$ & 11 & $27.5 \%$ & 1 & $14.3 \%$ & 0.659 \\
\hline Resection and end to end anastomosis & 21 & $44.7 \%$ & 18 & $45.0 \%$ & 3 & $42.9 \%$ & 1.000 \\
\hline resection with patch augmentation & 2 & $4.3 \%$ & 2 & $5.0 \%$ & 0 & $0.0 \%$ & 1.000 \\
\hline resection with Subclavian Flap & 8 & $17.0 \%$ & 6 & $15.0 \%$ & 2 & $28.6 \%$ & 0.585 \\
\hline \multirow{2}{*}{$\begin{array}{l}\text { subclavian flap and patch augmentation } \\
\text { PA Banding }\end{array}$} & 3 & $6.4 \%$ & 3 & $7.5 \%$ & 0 & $0.0 \%$ & 1.000 \\
\hline & 14 & $29.8 \%$ & 13 & $32.5 \%$ & 1 & $14.3 \%$ & 0.657 \\
\hline $\begin{array}{l}\text { Cross-clamp time }(\min ) \\
\text { Median }[\mathrm{IQR}] \\
\text { Range }\end{array}$ & $\begin{array}{r}19.0 \\
(1\end{array}$ & $\begin{array}{l}6.0-25.0] \\
0-59.0)\end{array}$ & 18. & $\begin{array}{l}15.5-23.5] \\
0-57.0)\end{array}$ & 22. & $\begin{array}{l}7.0-53.0] \\
-59.0)\end{array}$ & 0.104 \\
\hline
\end{tabular}

IQR: interquartile range; COA: coarctation of the aorta; PA: pulmonary artery

After surgery, patients were followed up during their in-hospital stay and follow-up visits were scheduled after discharge. The median duration of follow-up was 18 months (95\% CI=14 to 22 months). No mortality was recorded during hospital stay or later in the follow-up period.

Table (4) shows the details of postoperative period and mid-term outcomes. The reintervention group showed significantly longer durations of mechanical ventilation, inotrope administration, and ICU stay as well as higher mean postoperative PG and median PG at last follow-up visit. 
Table (4): Postoperative period and follow-up

\begin{tabular}{|c|c|c|c|c|c|c|c|}
\hline \multirow{2}{*}{ Postoperative inotrop } & \multicolumn{2}{|c|}{$\begin{array}{c}\text { Total } \\
(n=47)\end{array}$} & \multicolumn{2}{|c|}{$\begin{array}{l}\text { No reintervention } \\
\qquad(\mathrm{n}=\mathbf{4 0})\end{array}$} & \multicolumn{2}{|c|}{$\begin{array}{l}\text { Reintervention } \\
\quad(n=7)\end{array}$} & \multirow[t]{2}{*}{$\mathbf{P}$} \\
\hline & & & & & & & \\
\hline & 22 & $46.8 \%$ & 18 & $45.0 \%$ & 4 & $57.1 \%$ & 0.690 \\
\hline \multicolumn{8}{|c|}{ Duration of mechanical ventilation (days) } \\
\hline Median [IQR] & \multirow{2}{*}{\multicolumn{2}{|c|}{$\begin{array}{c}2.0[2.0-6.0] \\
(1.0-45.0)\end{array}$}} & \multirow{2}{*}{\multicolumn{2}{|c|}{$\begin{array}{c}2.0[2.0-5.0] \\
(1.0-30.0)\end{array}$}} & \multirow{2}{*}{\multicolumn{2}{|c|}{$\begin{array}{l}7.0[4.0-20.0] \\
\quad(2.0-45.0)\end{array}$}} & $0.010 *$ \\
\hline Range & & & & & & & \\
\hline \multicolumn{8}{|c|}{ Duration of inotrope administration (days) } \\
\hline Median [IQR] & \multicolumn{2}{|c|}{$\begin{array}{c}2.0[1.0-5.0] \\
(1.0-28.0)\end{array}$} & \multicolumn{2}{|c|}{$\begin{array}{l}2.0[1.0-3.5] \\
\quad(1.0-8.0)\end{array}$} & \multicolumn{2}{|c|}{$\begin{array}{c}6.0[4.0-12.0] \\
(2.0-28.0)\end{array}$} & $0.001 *$ \\
\hline \multicolumn{8}{|c|}{ ICU length of stay (days) } \\
\hline Median [IQR] & \multirow{2}{*}{\multicolumn{2}{|c|}{$\begin{array}{c}5.0[3.0-7.0] \\
(2.0-30.0)\end{array}$}} & \multirow{2}{*}{\multicolumn{2}{|c|}{$\begin{array}{c}4.5[3.0-6.0] \\
(2.0-17.0)\end{array}$}} & \multirow{2}{*}{\multicolumn{2}{|c|}{$\begin{array}{c}13.0[6.0-15.0] \\
(2.0-30.0)\end{array}$}} & $0.011^{*}$ \\
\hline Range & & & & & & & \\
\hline \multicolumn{8}{|c|}{ Postoperative LV function } \\
\hline Poor & 4 & $8.5 \%$ & 4 & $10.0 \%$ & 0 & $0.0 \%$ & 0.808 \\
\hline Moderate & 9 & $19.1 \%$ & 7 & $17.5 \%$ & 2 & $28.6 \%$ & \\
\hline Good & 34 & $72.3 \%$ & 29 & $72.5 \%$ & 5 & $71.4 \%$ & \\
\hline \multicolumn{8}{|c|}{ Postoperative ECHO PG } \\
\hline Mean \pm SD & \multirow{2}{*}{\multicolumn{2}{|c|}{$\begin{array}{l}21.1 \pm 10.9 \\
(5.0-58.0)\end{array}$}} & \multirow{2}{*}{\multicolumn{2}{|c|}{$\begin{array}{c}18.2 \pm 8.1 \\
(5.0-35.0)\end{array}$}} & \multirow{2}{*}{\multicolumn{2}{|c|}{$\begin{array}{c}37.7 \pm 10.4 \\
(30.0-58.0)\end{array}$}} & $<0.001 *$ \\
\hline Range & & & & & & & \\
\hline \multicolumn{8}{|c|}{ Postoperative complications } \\
\hline Yes & 29 & $61.7 \%$ & 23 & $57.5 \%$ & 6 & $85.7 \%$ & 0.225 \\
\hline Hypertension & 20 & $42.6 \%$ & 15 & $37.5 \%$ & 5 & $71.4 \%$ & 0.119 \\
\hline Infection & 4 & $8.5 \%$ & 3 & $7.5 \%$ & 1 & $14.3 \%$ & 0.488 \\
\hline Chylothorax & 3 & $6.4 \%$ & 2 & $5.0 \%$ & 1 & $14.3 \%$ & 0.391 \\
\hline Heart failure & 2 & $4.3 \%$ & 2 & $5.0 \%$ & 0 & $0.0 \%$ & 1.000 \\
\hline Pneumothorax & 2 & $4.3 \%$ & 1 & $2.5 \%$ & 1 & $14.3 \%$ & 0.278 \\
\hline Other complications & 5 & $10.6 \%$ & 2 & $5.0 \%$ & 3 & $42.9 \%$ & $0.019 *$ \\
\hline \multicolumn{8}{|c|}{$L V$ function at last follow-up } \\
\hline Poor & 1 & $2.1 \%$ & 1 & $2.5 \%$ & 0 & $0.0 \%$ & 1.000 \\
\hline Moderate & 3 & $6.4 \%$ & 3 & $7.5 \%$ & 0 & $0.0 \%$ & \\
\hline Good & 43 & $91.5 \%$ & 36 & $90.0 \%$ & 7 & $100.0 \%$ & \\
\hline \multicolumn{8}{|c|}{ ECHO PG at last follow-up } \\
\hline Median [IQR] & \multirow{2}{*}{\multicolumn{2}{|c|}{$\begin{array}{c}12.0[0.0-18.0] \\
\quad(0.0-26.0)\end{array}$}} & \multirow{2}{*}{\multicolumn{2}{|c|}{$\begin{array}{c}11.0[0.0-17.5] \\
\quad(0.0-23.0)\end{array}$}} & \multirow{2}{*}{\multicolumn{2}{|c|}{$\begin{array}{c}15.0[13.0-25.0] \\
(0.0-26.0)\end{array}$}} & $0.042 *$ \\
\hline Range & & & & & & & \\
\hline
\end{tabular}

IQR: interquartile range; SD: standard deviation; *: significant difference.

Table (5) shows the results of Cox proportional hazard regression analysis that was performed to identify potential risk factors for reintervention. Only postoperative PG contributed significantly to the need for reintervention.

Table (5): Logistic regression analysis to identify risk factors of reintervention

\begin{tabular}{lcccc}
\hline & P & Adjusted HR & \multicolumn{2}{c}{ 95\% CI for HR } \\
& & & 0.025 & Upper \\
\hline Birth weight $(\mathrm{Kg})$ & 0.247 & 0.256 & 2.572 \\
Prostaglandin administration & 0.942 & 84837.529 & 0.000 & $1.345 \mathrm{E}+138$ \\
Postoperative inotropes use duration (days) & 0.939 & 0.996 & 0.893 & 1.110 \\
Postoperative pressure gradient & $0.031^{*}$ & 1.068 & 1.006 & 1.133 \\
\hline
\end{tabular}

CI: confidence interval; HR: hazard ratio; *: significant difference.

Figure (2) demonstrates ROC curve for postoperative PG showing excellent discrimination (AUC $=0.948$ ) with an optimal cut-off value $>25$. 


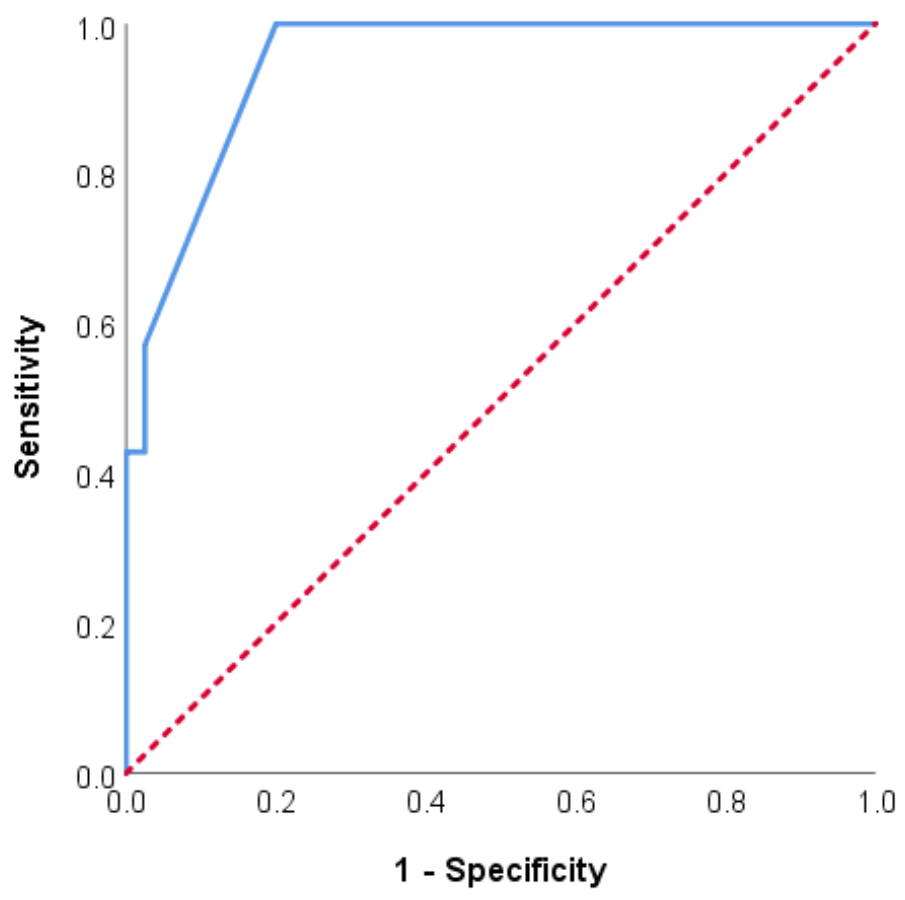

Figure (2): ROC curve for postoperative pressure gradient as predictor for reintervention. Area under the curve $=0.948(95 \%$ CI: 0.841 to $0.992, p<0.001)$; cut-off value $>25,100 \%$ sensitivity, $80 \%$ specificity, $46.7 \%$ positive predictive value, and $100 \%$ negative predictive value.

\section{DISCUSSION}

The present study aimed to evaluate the rate and predictors for reintervention after surgical coarctation repair by different techniques through left thoracotomy. Forty-seven patients with CoA were categorized according to their need for reintervention with catheter angiographic-guided balloon dilatation into two groups. Only 7 patients required reintervention (14.9\%). The rates reported by previous studies widely varied, ranging from 5.9 to $46.6 \%{ }^{(\mathbf{1 8 - 2 2})}$.

We compared patients' characteristics as well as preoperative and postoperative clinical and echocardiographic PG assessments between patients requiring reintervention and those who did not.

Age at first repair did not differ significantly between the two groups in the present study, though the median age was younger in patients who required reintervention as all of them were less than 15 days old. This tendency of patients with CoA to have younger age, with the results not reaching statistical significance, was also reported by Adamson et al. ${ }^{(13)}$, Burch et al. (14), and Liang et al. (23). However, McElhinney et al. ${ }^{(17)}$ reported that age $<15$ days at surgery was associated with a higher risk of recoarctation (OR: 6.3, 95\% CI $=1.77-22.4, \mathrm{p}=0.005)$. Moreover, Lehnert et al. ${ }^{(9)}$ found that reintervention was significantly associated with an age at surgery below 15 days (OR: 0.98, 95\% CI: 0.97-1.00, $\mathrm{p}=0.034$ ).

Comparison of body weight at birth and at surgery between the two studied groups showed a significant association of birth weight $<2.5 \mathrm{Kg}$ only with reintervention. However, multivariate analysis using Cox proportional hazard regression did not show significant association. Truong et al. ${ }^{(19)}$ found that those who developed recoarctation had significantly lower median birth weight ( 3.16 vs $2.26 \mathrm{~kg}, \mathrm{p}=0.04$ ). As regards weight at surgery, the observed lack of significant impact on reintervention was similarly stated by several previous studies $\mathbf{( 9 , 1 3 , 1 4 , 1 7 , 1 9 , 2 3 )}$. On the contrary, low weight at surgery represented a risk factor (OR: 0.016, $95 \%$ CI: 0.001-0.51, p = 0.047) in a study by Soynov et al. ${ }^{(18)}$. In addition, some previous studies $(14,17,24,25)$ found that birth weight and weight at surgery were not associated with a higher risk of recoarctation.

Our results revealed the lack of association between CoA and the presence of associated cardiac anomalies, which is in accordance with other studies (9,12,13,17,19, 24, 26). However, aortic arch hypoplasia was reported as a risk factor by Hager $\boldsymbol{e t}$ al. ${ }^{(16)}$ (OR: 2.864, 95\% CI: 1.581-5.186, $\mathrm{p}=0.001$ ).

Preoperative administration of prostaglandin E1 is advocated in some cases to ameliorate clinical manifestations of CoA by reopening of the ductus arteriosus or the aortic isthmus until the time of surgery $(27,28)$. All patients who required reintervention in our cohort were administered prostaglandins preoperatively. Nevertheless, multivariate analysis showed the lack of significant contribution to the risk of reintervention. Preoperative prostaglandin E1 administration was identified by Lehnert $\boldsymbol{e t}$ al. ${ }^{(9)}$ as a risk factor of recoarctation (OR: 1.53, 95\% CI: 0.902.60, $\mathrm{p}=0.004)$. Advantages of prostaglandin administration in neonates with CoA awaiting surgery include reduction of preoperative mortality, lowering of systemic resistance, and relaxation of ductus arteriosus or the aortic arch ${ }^{(29,30)}$. On the other hand, Burch et al. 
${ }^{(14)}$ and McElhinney et al. ${ }^{(17)}$ did not find a significant association with recoarctation.

We did not find a significant association between the type of surgery and reintervention. The type of surgical repair was assessed as a risk factor for recoarctation and reintervention in previous studies, with conflicting results. Patch aortoplasty was reported to be associated with recoarctation by two studies $(\mathbf{1 3}, \mathbf{3 1}$. One study ${ }^{(19)}$ associated extended end-to-end repair anastomosis with increased risk of recoarctation. Three studies identified repair by balloon angioplasty as a risk factor for recoarctation ${ }^{(20,21,32)}$.

In the current study, patients with reintervention had significantly higher mean postoperative PG. On multivariate regression, postoperative PG was the only independent risk factor that impacted the risk of reintervention significantly. We identified an optimal cut-off value $>25$ to predict the risk of reintervention, with $100 \%$ negative predictive value indicating that the test can be used to exclude cases that will not require reintervention. A plausible explanation for the increased postoperative PG on echocardiographic assessment is that it is caused by residual arch obstruction. In accordance with this finding, Truong et al. ${ }^{(19)}$ reported that those who developed recoarctation had significantly higher postoperative peak Doppler velocity ( $2.55 \mathrm{vs} .1 .80 \mathrm{~m} / \mathrm{s}$, $\mathrm{p}=0.01)$ compared with those who did not, with AUC $=0.728(\mathrm{p}=0.01)$ and a cut-off value of $2.12 \mathrm{~m} / \mathrm{s} \mathrm{had} \mathrm{a}$ sensitivity of $63 \%$ and a specificity of $83 \%$.

The present study was subject to some limitations, including the small sample size, which may result in failure to detect statistical significance for some variables, though clinical significance may be apparent from summary statistics. Recoarctation may occur few years after the primary repair and thus a longer follow-up period may reveal a higher incidence of reintervention and elucidate the role of some potential risk factors.

\section{CONCLUSION}

Repair of CoA through thoracotomy has an overall good outcome. However, reintervention is required in some patients and elevated predischarge pressure gradient on echocardiographic assessment $<25$ can exclude recoarctation.

\section{REFERENCES}

1. Kenny D, Hijazi $Z$ (2011): Coarctation of the aorta: from fetal life to adulthood. Cardiology Journal, 18(5):487-95.

2. Beekman R (2013): Coarctation of the aorta. In: Allen HD, Driscoll DJ, Shaddy RE, Feltes TF, editors. Moss and Adams' heart disease in infants, children, and adolescents. Philadelphia: Wolters Kluwer Health, Pp. 1044-60.

3. Ho S, Anderson $R$ (1979): Coarctation, tubular hypoplasia, and the ductus arteriosus. Histological study of 35 specimens. British Heart Journal, 41(3):268-74.
4. Price T, Whisenhunt A, Policha A et al. (2014): Middle aortic coarctation. Annals of Vascular Surgery, 28(5):1314.e15-21.

5. Anderson R, Lenox C, Zuberbuhler J (1983): Morphology of ventricular septal defect associated with coarctation of aorta. British Heart Journal, 50(2):176-81.

6. Becker A, Becker M, Edwards J (1970): Anomalies associated with coarctation of aorta: particular reference to infancy. Circulation, 41(6):1067-75.

7. Rosenthal E (2005): Coarctation of the aorta from fetus to adult: curable condition or life long disease process? Heart (British Cardiac Society), 91(11):1495-502.

8. Ward K, Pryor R, Matson J et al. (1990): Delayed detection of coarctation in infancy: implications for timing of newborn follow-up. Pediatrics, 86(6):972-6.

9. Lehnert A, Villemain O, Gaudin R et al. (2019): Risk factors of mortality and recoarctation after coarctation repair in infancy. Interactive Cardiovascular and Thoracic Surgery, 29(3):469-75.

10. Kappetein A, Zwinderman A, Bogers A et al. (1994): More than thirty-five years of coarctation repair. An unexpected high relapse rate. The Journal of Thoracic and Cardiovascular Surgery, 107(1):87-95.

11. Backer C, Paape K, Zales V et al. (1995): Coarctation of the aorta. Repair with polytetrafluoroethylene patch aortoplasty. Circulation, 92(9):132-6.

12. Kaushal S, Backer C, Patel J et al. (2009): Coarctation of the aorta: midterm outcomes of resection with extended end-to-end anastomosis. Ann Thorac Surg., 88(6):1932-8.

13. Adamson G, Karamlou T, Moore $P$ et al. (2017): Coarctation index predicts recurrent aortic arch obstruction following surgical repair of coarctation of the aorta in infants. Pediatric Cardiology, 38(6):1241-6.

14. Burch P, Cowley C, Holubkov $R$ et al. (2009): Coarctation repair in neonates and young infants: is small size or low weight still a risk factor? The Journal of Thoracic and Cardiovascular Surgery, 138(3):547-52.

15. Dias M, Barros A, Leite-Moreira A et al. (2020): Risk factors for recoarctation and mortality in infants submitted to aortic coarctation repair: A systematic review. Pediatric Cardiology, 41(3):561-75.

16. Hager A, Schreiber C, Nutzl S et al. (2009): Mortality and restenosis rate of surgical coarctation repair in infancy: a study of 191 patients. Cardiology, 112(1):3641.

17. McElhinney D, Yang S, Hogarty A et al. (2001): Recurrent arch obstruction after repair of isolated coarctation of the aorta in neonates and young infants: is low weight a risk factor? The Journal of Thoracic and Cardiovascular Surgery, 122(5):883-90.

18. Soynov I, Sinelnikov Y, Gorbatykh Y et al. (2018): Modified reverse aortoplasty versus extended anastomosis in patients with coarctation of the aorta and distal arch hypoplasia. European Journal of CardioThoracic Surgery: Official Journal of the European Association for Cardio-Thoracic Surgery, 53(1):254-61.

19. Truong $D$, Tani $L$, Minich $L$ et al. (2014): Factors associated with recoarctation after surgical repair of coarctation of the aorta by way of thoracotomy in young infants. Pediatric Cardiology, 35(1):164-70.

20. Chiu H, Wang J, Chen Y et al. (2013): Long-term outcomes of native coarctation of the aorta after balloon angioplasty or surgical aortoplasty in newborns and 
young infants less than 3 months of age. Acta Cardiologica Sinica, 29(2):168-74.

21. Sen S, Garg S, Rao S et al. (2018): Native aortic coarctation in neonates and infants: Immediate and midterm outcomes with balloon angioplasty and surgery. Annals of Pediatric Cardiology, 11(3):261-6.

22. Tulzer A, Mair R, Kreuzer M et al. (2016): Outcome of aortic arch reconstruction in infants with coarctation: Importance of operative approach. The Journal of Thoracic and Cardiovascular Surgery, 152(6):1506-13.

23. Liang C, Su W, Chung $\mathrm{H}$ et al. (2009): Balloon angioplasty for native coarctation of the aorta in neonates and infants with congestive heart failure. Pediatrics and Neonatology, 50(4):152-7.

24. Kumar T, Zurakowski D, Sharma $R$ et al. (2011): Prediction of recurrent coarctation by early postoperative blood pressure gradient. The Journal of Thoracic and Cardiovascular Surgery, 142(5):1130-6.

25. Pearl J, Manning P, Franklin C et al. (2004): Risk of recoarctation should not be a deciding factor in the timing of coarctation repair. The American Journal of Cardiology, 93(6):803-5.

26. Welke K, Diggs B, Karamlou T et al. (2009): Comparison of pediatric cardiac surgical mortality rates from national administrative data to contemporary clinical standards. Ann Thorac Surg., 87(1):216-22.
27. Reddy V, McElhinney D, Sagrado T et al. (1999): Results of 102 cases of complete repair of congenital heart defects in patients weighing 700 to 2500 grams. The Journal of Thoracic and Cardiovascular Surgery, 117(2):324-31.

28. Freed M, Heymann M, Lewis A et al. (1981): Prostaglandin E1 infants with ductus arteriosusdependent congenital heart disease. Circulation, 64(5):899-905.

29. Leoni F, Huhta J, Douglas J et al. (1984): Effect of prostaglandin on early surgical mortality in obstructive lesions of the systemic circulation. British Heart Journal, 52(6):654-9.

30. Lewis A, Freed M, Heymann M et al. (1981): Side effects of therapy with prostaglandin E1 in infants with critical congenital heart disease. Circulation, 64(5):8938 .

31. Quaegebeur J, Jonas R, Weinberg A et al. (1994): Outcomes in seriously ill neonates with coarctation of the aorta. A multiinstitutional study. The Journal of Thoracic and Cardiovascular Surgery, 108(5):841-51.

32. Fiore A, Fischer L, Schwartz T et al. (2005): Comparison of angioplasty and surgery for neonatal aortic coarctation. Ann Thorac Surg., 80(5):1659-64. 